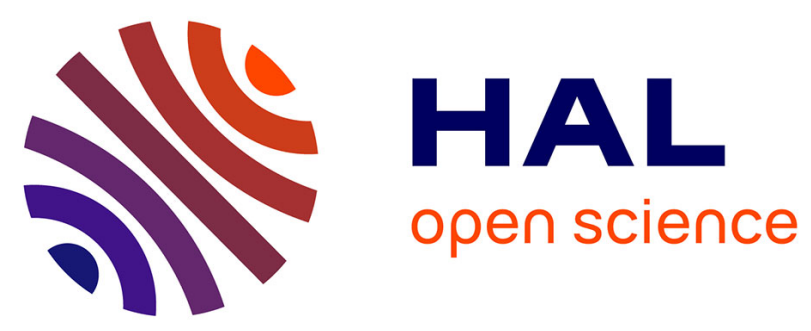

\title{
Stability Analysis of a Metabolic Model with Sequential Feedback Inhibition
}

\author{
Yacine Chitour, Frédéric Grognard, Georges Bastin
}

\section{To cite this version:}

Yacine Chitour, Frédéric Grognard, Georges Bastin. Stability Analysis of a Metabolic Model with Sequential Feedback Inhibition. Luca Benvenuti, Alberto De Santis, Lorenzo Farina. Positive Systems: Proceedings of the First Multidisciplinary International Symposium on Positive Systems: Theory and Applications (POSTA 2003), 294, Springer Berlin Heidelberg, pp.143-150, 2003, 10.1007/978-3-54044928-7_20.hal-01091656

\section{HAL Id: hal-01091656 \\ https://inria.hal.science/hal-01091656}

Submitted on 5 Dec 2014

HAL is a multi-disciplinary open access archive for the deposit and dissemination of scientific research documents, whether they are published or not. The documents may come from teaching and research institutions in France or abroad, or from public or private research centers.
L'archive ouverte pluridisciplinaire HAL, est destinée au dépôt et à la diffusion de documents scientifiques de niveau recherche, publiés ou non, émanant des établissements d'enseignement et de recherche français ou étrangers, des laboratoires publics ou privés. 


\title{
Stability analysis of a metabolic model with sequential feedback inhibition
}

\author{
Y. Chitour \\ Département de Mathématiques \\ F. Grognard \\ Projet COMORE - INRIA \\ Université de Paris sud \\ BP 9306902 Sophia-Antipolis Cedex \\ Bâtiment 425 \\ 91405 Orsay, France \\ Email : Yacine.Chitour@math.u-psud.fr \\ G. Bastin \\ Centre for Systems Engineering and Applied Mechanics(CESAME) \\ Université Catholique de Louvain \\ Bâtiment Euler,4-6, avenue G.Lemaitre, \\ 1348 Louvain la Neuve, Belgium \\ Email : bastin@auto.ucl.ac.be
}

Email : Frederic.Grognard@sophia.inria.fr

\begin{abstract}
:
This paper deals with the stability analysis of a simple metabolic system with feedback inhibition. The system is a sequence of monomolecular enzymatic reactions. The last metabolite acts as a feedback regulator for the first enzyme of the pathway. The enzy matic reactions of the pathway satisfy MichaelisMenten kinetics. The inhibition is described by an hyperbolic model. Without inhibition, it is clear that the system is cooperative and has a single globally asymptotically stable equilibrium. But, in the common situation where there is inhibition, the system is no longer cooperative and the stability analysis is more intricate. In this paper we exhibit sufficient conditions on the kinetic parameters in order to guarantee that this simple metabolic system with inhibition still has a single globally asymptotically stable equilibrium.
\end{abstract}




\section{Introduction}

The huge set of biochemical reactions which occur inside living cells is called the Cellular Metabolism. It is usually represented by an intricate network connecting the involved biochemical species (called "metabolites"). The pathways of the network are called "metabolic pathways". In the metabolic engineering literature, it is widely accepted that" despite their immense complexity, metabolic systems are characterized by their ability to reach stable steady states" (quoted from [6], Chapter 4). It should however be fair torecognize that a mathematical analysis of this fundamental stability property is a difficult question which was not much investigated. Our objective in this paper is to provide a modest contribution to this question. We shall limit ourselves to simple metabolic pathways which are made up of a sequence of mono-molecular enzyme-catalysed reactions as where $X_{i}(i=1, \cdots, n)$ represent the successive metabolites of the pathway:

$$
X_{1} \rightarrow X_{2} \rightarrow \cdots \rightarrow X_{n}
$$

A typical situation is when such a simple pathway is located between two branch points of a complex metabolic network. We shall consider the case of a so-called sequential feedback inhibition (cf. [6]) where the last metabolite $X_{n}$ acts as an inhibitor of the first reaction $X_{1} \longrightarrow X_{2}$. This inhibition is represented by the dotted feedback arrow in Fig.1. The velocity of each enzymatic reaction $X_{i} \longrightarrow$ $X_{i+1}$ is represented by a Michaelis-Menten kinetic function :

$$
\varphi_{i}\left(x_{i}\right)=\frac{a_{i} x_{i}}{k_{i}+x_{i}}
$$

where $x_{i}$ denotes the intracellular molar fraction of the metabolite $X_{i}, a_{i}$ is the maximal velocity and $k_{i}$ the so-called half-saturation constant. It is assumed that the velocity of the first reaction $X_{1} \longrightarrow X_{2}$ is inhibited by the last metabolite with a multiplicative hyperbolic inhibition function of the form:

$$
\psi_{\alpha}\left(x_{n}\right)=\frac{1}{1+\alpha x_{n}}
$$

In addition, it is assumed that the cell metabolism is analysed during a period of exponential cell growth with a constant specific growth rate $\mu$. Under these assumptions and notations, a mass balance dynamical model is formulated as:

$$
(\Sigma)\left\{\begin{array}{l}
\dot{x}_{1}=-\frac{a_{1} x_{1}}{\left(k_{1}+x_{1}\right)\left(1+\alpha x_{n}\right)}-\mu x_{1}+c, \\
\dot{x}_{2}=\frac{a_{1} x_{1}}{\left(k_{1}+x_{1}\right)\left(1+\alpha x_{n}\right)}-\frac{a_{2} x_{2}}{\left(k_{2}+x_{2}\right)}-\mu x_{2}, \\
\dot{x}_{i}=\frac{a_{i-1} x_{i-1}}{\left(k_{i-1}+x_{i-1}\right)}-\frac{a_{i} x_{i}}{\left(k_{i}+x_{i}\right)}-\mu x_{i}, 3 \leq i \leq n,
\end{array}\right.
$$


where $n \geq 3$ is a positive integer, $x=\left(x_{1}, \cdots, x_{n}\right)^{T} \in \mathbb{R}^{n}$, and all the $a_{i}, k_{i}$ 's, $c, \alpha, \mu$ are positive constants.

In this model $c$ denotes the inflow rate of the first metabolite $X_{1}$ of the sequence and is assumed to be constant. Without inhibition (i.e. $\alpha=0$ ), the system $\Sigma$ is clearly compartmental and cooperative which implies that it has a single globally asymptotically stable equilibrium. But if there is inhibition, the system is no longer cooperative and the stability analysis is more difficult. Our contribution in this paper will be to exhibit sufficient conditions on the kinetic parameters that guarantee that the simple metabolic system $\Sigma$ with feedback inhibition still has a single globally asymptotically stable equilibrium.

\section{Notations and statement of the theorem}

\section{$2.1 \quad$ Notations}

Consider the metabolic system $(\Sigma)$ with feedback inhibition (1. 3). Up to a change of variable (the $x_{i}$ 's are multiplied by $\alpha$ ) and a time reparameterization (the time is multiplied by $\mu$ ), we may assume that $\mu=\alpha=1$ and the model is rewritten:

$$
(\Sigma)\left\{\begin{array}{l}
\dot{x}_{1}=-\varphi_{1}\left(x_{1}\right) \psi\left(x_{n}\right)-x_{1}+c \\
\dot{x}_{2}=\varphi_{1}\left(x_{1}\right) \psi\left(x_{n}\right)-\varphi_{2}\left(x_{2}\right)-x_{2}, \\
\dot{x}_{i}=\varphi_{i-1}\left(x_{i-1}\right)-\varphi_{i}\left(x_{i}\right)-x_{i}, 3 \leq i \leq n
\end{array}\right.
$$

where $\varphi_{i}$ is defined in (1. 1) and $\psi:=\psi_{1}$ in (1. 2). In this model, $c$ stands for $\frac{\alpha c}{\mu}$ (with the original $c$ in that last formula) and similarly, for $1 \leq i \leq n, a_{i}$ stands for $\frac{\alpha a_{i}}{\mu}$ and $k_{i}$ for $\alpha k_{i}$.

We introduce some notations: for $2 \leq i \leq n, f_{i}(x)=x+\varphi_{i}(x)$ and $f_{1, x_{n}}(x)=x+\psi\left(x_{n}\right) \varphi_{1}(x)$. It is clear that the $\varphi_{i}$ 's, $1 \leq i \leq n$, are strictly monotone functions on $R_{+}$and realize bijections between $\mathbb{R}_{+}$and $\left[0, a_{i}\right)$. We use $\varphi_{i}^{-1}$ to denote the inverse function. For $2 \leq i \leq n$, the $f_{i}$ 's are strictly monotone functions on $\mathbb{R} R_{+}$and realize bijections from $\mathbb{R} R_{+}$to $\mathbb{R} R_{+} ; f_{i}^{-1}$ denotes the inverse function of $f_{i}$ and $g_{i}=\varphi_{i} \circ f_{i}^{-1}$. Let $M: \mathbb{R}_{+} \rightarrow \mathbb{R}_{+}$by $M=f_{n}^{-1} \circ g_{n-1} \circ \cdots \circ g_{2}$.

For every $x \geq 0$ and $2 \leq i \leq n$, we have

$$
1<f_{i}^{\prime}(x)=1+\varphi_{i}^{\prime}(x) \leq 1+\frac{a_{i}}{k_{i}}, \frac{k_{i}}{a_{i}+k_{i}} \leq\left(f_{i}^{-1}\right)^{\prime}(x)=\frac{1}{1+\varphi_{i}^{\prime}\left(f_{i}^{-1}(x)\right)}<1 .
$$

Note that the $f_{i}$ 's, the $\varphi_{i}$ 's are concave functions on $\mathbb{R}_{+}$(negative second derivative) for $2 \leq i \leq n$. 
This implies that the $g_{i}$ 's are also concave. Therefore, we have for $2 \leq i \leq n$ and every $x \geq 0$

$$
0<g_{i}^{\prime}(x)=\frac{\varphi_{i}^{\prime}\left(f_{i}^{-1}(x)\right)}{1+\varphi_{i}^{\prime}\left(f_{i}^{-1}(x)\right)} \leq g_{i}^{\prime}(0)=\frac{a_{i}}{k_{i}+a_{i}}
$$

and since

$$
M^{\prime}(x)=\left(f_{i}^{-1}\right)^{\prime}\left(g_{n-1} \circ \cdots \circ g_{2}(x)\right)\left[\prod_{i=3}^{n-1} g_{i}^{\prime}\left(g_{i-1} \circ \cdots \circ g_{2}(x)\right)\right] g_{2}^{\prime}(x),
$$

we can then conclude from (2. 2) and (2.3) that, for every $x \geq 0$

$$
0<M^{\prime}(x)<\prod_{i=2}^{n-1} \frac{a_{i}}{k_{i}+a_{i}}
$$

As for $f_{1, x_{n}}$, for every $x_{n} \in \mathbb{R}_{+}$, it behaves like any $f_{i}, 2 \leq i \leq n$. Define $z: \mathbb{R}_{+} \rightarrow[0, c)$ by $z(b)=f_{1, b}^{-1}(c)$. Later we will study in more details that application. Let $F$ be the vector field on $I R^{n}$ simply defined by the right-hand side of $(\Sigma)$. Let $K=\mathbb{I} R_{+}^{n}$ the non-negative orthant and $K_{+}$the positive orthant. The positive cone $K$ defines a closed partial order relation $\leq$ on $\mathbb{R}^{n}$ defined by $x \leq y$ if and only if $y-x \in K$. It means that $x_{i} \leq y_{i}$ holds for every $1 \leq i \leq n$. We write $x<y$ if $x \leq y$ and $x \neq y$, and $x \ll y$ whenever $y-x \in \operatorname{Int}(K)=K_{+}$. This notation extends trivially to subsets of $\mathbb{R}^{n}$. Moreover, if $x \leq y$, then the set $P_{x, y}=\left\{z \in \mathbb{R}^{n}, \quad x \leq z \leq y\right\}$ is a parallellepiped. Let $v \in K_{+}$ defined by $v=(1, \cdots, 1)$.

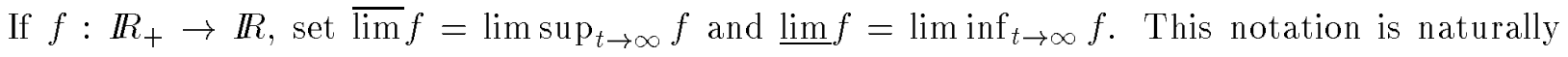
extended to the vectorial case using the partial order defined previously. We will also consider sometimes the function $V: \mathbb{R}^{n} \rightarrow \mathbb{R}$ defined by $V(x)=\sum_{i=1}^{n} x_{i}$. Let $T_{d}, d>0$, be the simplex of $K$ defined as the the set of $x \in K$ so that $V(x) \leq d$.

If $x, y \in X$, then $[x, y]$ denotes the segment with extremities $x$ and $y$, i.e. the set of points $t x+(1-t) y$ for $t \in[0,1]$. A set $X \subset \mathbb{R}^{n}$ is said to be p-convex if for every $x, y \in X$ with $x \leq y$ then $[x, y] \subset X$. Let $m$ be a positive integer. An $m \times m$ matrix $A=\left(a_{i j}\right)$ is said to be irreductible if for every nonempty, proper subset $I \subset\{1, \cdots, n\}$, there is an $i \in I$ and $j \in\{1, \cdots, n\} / I$ such that $a_{i j} \neq 0$. There is a graph-theoretic formulation of irreducibility (cf. [5]): consider the directed graph $\mathcal{G}$ whose set of vertices is $\{1, \cdots, n\}$; two vertices $i, j$ have a directed edge from $i$ to $j$ if $a_{i j} \neq 0$. Then $A$ is irreducible if its directed graph $\mathcal{G}$ is connected.

A dynamical system $(G)$ given by $\dot{x}=G(x), x \in D$ with $D$ open, $G: D \rightarrow \mathbb{R}^{n}$ of class $C^{1}$ is said to be cooperative (see [5]) if, for every $x \in D, 1 \leq i, j \leq n$ and $i \neq j$,

$$
\frac{\partial G_{i}}{\partial x_{j}} \geq 0
$$


If in addition the jacobian matrix $D G(x)$ is irreducible for every $x \in D$, then $(G)$ is said to be irreducible cooperative.

Remark 2. 1 It is worth noticing that $(\Sigma)$ is not cooperative with respect to the partial order defined by $K$. It should be pointed out that the coefficient in the jacobian $D F(x)$ that renders $(\Sigma)$ non cooperative is $\frac{\partial f_{2}}{\partial x_{n}}$, which is indeed negative.

We will consider auxiliary systems $(\Sigma)_{b}, b \geq 0$, given by

$$
(\Sigma)_{b}\left\{\begin{array}{l}
\dot{x}_{1}=-\varphi_{1}\left(x_{1}\right) \psi\left(x_{n}\right)-x_{1}+c \\
\dot{x}_{2}=\varphi_{1}\left(x_{1}\right) \psi(b)-\varphi_{2}\left(x_{2}\right)-x_{2} \\
\dot{x}_{k}=\varphi_{k-1}\left(x_{k-1}\right)-\varphi_{k}\left(x_{k}\right)-x_{k}, 3 \leq k \leq n
\end{array}\right.
$$

where the difference with $(\Sigma)$ lies in the equation defining $\dot{x}_{2}$ : the variable $x_{n}$ is frozen at the constant value $b$. We use $F_{b}(x)$ to denote the right-hand side of $(\Sigma)_{b}$. Now, the $(2, n)$-coefficient in $D F_{b}(x)$ is identically equal to zero. If $x \in K$, we use $\gamma_{x}, \gamma_{x}^{b}$ respectively, to denote the trajectory of $(\Sigma),(\Sigma)_{b}$ respectively, which starts at $x$.

\subsection{Preliminary results and statement of the theorem}

Proposition 2. 2 The system $(\Sigma)$ has the following properties:

(1) ( $\Sigma$ ) has a unique equilibrium point $\bar{x} \in K_{+}$;

(2) For every $x \in K$ and every $t>0, \gamma_{x}(t) \in K_{+}$i.e. $K$ is a positively invariant set for $(\Sigma)$;

(3) For every $x \in K, \varlimsup \lim V\left(\gamma_{x}\right) \leq c$; i.e. $T_{c}$ is a global attractor of all the trajectories starting in $K$.

Remark 2. 3 Eventhough $(\Sigma)$ is not cooperative, it has some of the basic features that are required for the investigation of the $\omega$-limit sets of cooperative systems: an invariant cone with a repelling boundary, a bounded attractor and a unique equilibrium point.

Proof of Proposition 2. 2: we start with the argument of (1). Let $\bar{x} \in K$ an equilibrium point of $(\Sigma)$, i.e. $F(\bar{x})=0$. For $3 \leq k \leq n$, we have $\bar{x}_{k}=f_{k}^{-1} \circ \varphi_{k-1}\left(\bar{x}_{k-1}\right)$. In addition, we deduce from

$$
c-\bar{x}_{1}=\varphi\left(\bar{x}_{1}\right) \psi\left(\bar{x}_{n}\right)=f_{2}\left(\bar{x}_{2}\right),
$$


that $\bar{x}_{2}=f_{2}^{-1}\left(c-\bar{x}_{1}\right)$. Then $\bar{x}_{n}=M\left(c-\bar{x}_{1}\right)$. Therefore, since $M$ is strictly increasing, $(\Sigma)$ admits as many equilibrium points in $K$ as solutions in $[0, c]$ of the equation

$$
c=x+\varphi_{1}(x) \psi(M(c-x)) .
$$

Define $J:[0, c] \rightarrow \mathbb{R}_{+}$by $J(x)=x+\varphi_{1}(x) \psi(M(c-x))$. Since $\psi$ is strictly decreasing, so is $J$. Moreover $J(0)=0$ and $J(c)=c+\varphi_{1}(c)>c$. Then $J$ takes the value $c$ exactly once, which implies that equation (2.6) has exactly one solution in $(0, c)$. Claim $(1)$ is proved.

To establish (2), it is enough to show that $\partial K$, the boundary of $K$ is repelling for $(\Sigma)$. Let $y=\left(y_{1}, \cdots, y_{n}\right)^{T} \in \partial K$ and $x=\gamma_{y}$. Let $i_{0}=\max \left\{i, y_{i}=0\right\}$. If $i_{0}=1$, then $\dot{x}_{1}(0)>0$ and $x_{1}>0$ in a neighborhood of $t=0$ and we are done.

Assume now that $i_{0}>1$. If $y_{i_{0}-1}>0$, then $\dot{x}_{i_{0}}(0)>0$ and the result follows. If not, then $y_{i_{0}-1}=\dot{x}_{i_{0}}(0)=0$. The next alternative is whether $i_{0}-1=1$ or not. If $i_{0}-1=1$, then $\dot{x}_{i_{0}-1}(0)>0$ and $\ddot{x}_{i_{0}}(0)>0$; again the conclusion holds in this case. If $i_{0}-1>1$, we have a new alternative, whether $y_{i_{0}-2}>0$ or not. If $y_{i_{0}-2}>0$, then $\dot{x}_{i_{0}-1}(0)>0$, case already studied. If $y_{i_{0}-2}=0$, then $y_{i_{0}-l}=0$ for $l=0,1,2$. Proceeding in a similar manner, one can show that the last case to be treated corresponds to $y_{i_{0}-l}=0$ for $l=0, \cdots, i_{0}$. It implies that $x_{i_{0}}^{(l)}=0$ for $l=0, \cdots, i_{0}-1$. Since, anyhow $\dot{x}_{1}(0)>0$, we must have $\dot{x}_{i_{0}}^{\left(i_{0}\right)}>0$ and we are done.

Along trajectories of $(\Sigma)$, the derivative of $V$ verifies

$$
\dot{V}=c-V-\varphi_{n}\left(x_{n}\right)
$$

Therefore, if $x=\gamma_{x}(0) \in K$ and taking into account $(2)$, then $\varphi_{n}\left(\gamma_{x, n}(t)\right) \geq 0$. Then, $\dot{V} \leq c-V$, which clearly implies (3).

Remark 2. 4 At the light of Proposition 2. 2, the relevance of the auxiliary systems $(\Sigma)_{b}$ for understanding the dynamics of $(\Sigma)$ can be put forward. It is based on the two following remarks:

(a) for every $b \geq 0,(\Sigma)_{b}$ is an irreducible cooperative system (use the graph-theoretic formulation of irreducibility). This easily implies that $(\Sigma)_{b}$ verifies (2) (cf. Theorem 1.1 p.56 of [5]) and (3) (with possibly another positive constant instead of $c$ ). In fact $(\Sigma)_{b}$ is a hypercycle for which a Poincaré-Bendixon theory was developped for the compact $\omega$-limit sets of $(\Sigma)_{b}$, regardless of the dimension of the system(cf. [4]). Then, we expect taking advantage of the many deep results relative to that class of irreducible cooperative systems (for an excellent reference, $\mathrm{cf}[5]$ ). 
(b) for every $x \in K$ and $0 \leq b_{0}<b_{1}$, we have

$$
b_{0} \leq x_{n} \leq b_{1} \Rightarrow F_{b_{1}}(x) \leq F(x) \leq F_{b_{0}}(x)
$$

and, if $x_{1}>0$, then $\leq$ can be replaced everywhere by $<$ in the above equation. The monotonicity property expressed in (2.8) translates to the trajectories of $F$ and $F_{b}$ as explained next. Assume that we have shown the existence of $0 \leq b_{0}<b_{1}$ such that for every $x \in K$, there is some $t_{x}>0$ for which

$$
b_{0} \leq x_{n}(t) \leq b_{1} \text {, if } t \geq t_{x} .
$$

(This is clearly the case by (3) of Proposition 2. 2.) Using (2. 8), we have, for $t \geq t_{x}$,

$$
F_{b_{1}}\left(\gamma_{x}(t)\right) \leq F\left(\gamma_{x}(t)\right) \leq F_{b_{0}}\left(\gamma_{x}(t)\right)
$$

Set $y_{x}=\gamma_{x}\left(t_{x}\right)$. Since $F_{b}$ is a function of type $K$, we can apply a standard theorem of comparaison for differential inequalities ( $c f$. for instance Theorem 10 p.29 of [2]): for $t \geq t_{x}$,

$$
\gamma_{y_{x}}^{b_{1}}\left(t-t_{x}\right) \leq \gamma_{x}(t) \leq \gamma_{y_{x}}^{b_{0}}\left(t-t_{x}\right)
$$

Assume now that, according to Part $(a)$, the $\omega$-limit sets of $(\Sigma)_{b_{0}}$ and $(\Sigma)_{b_{1}}$ are investigated in details and one is able to show e.g. that every trajectory of $(\Sigma)_{b_{0}}\left((\Sigma)_{b_{1}}\right.$ respectively) starting in $K$ converges to $x^{b_{0}}$ ( $x^{b_{1}}$ respectively). In addition, assume that $b_{0} \ll x^{b_{0}}$ and $x^{b_{1}} \ll b_{1}$. Then the pair $\left(x_{n}^{b_{0}}, x_{n}^{b_{1}}\right)$ can be used in (2. 9) instead of $\left(b_{0}, b_{1}\right)$ in the bounding process for $\gamma_{x}(t)$ described above. If that procedure can be reproduced, one may hope to get more and more precise information on the $\omega$-limit sets of $(\Sigma)$. It is even tempting to conjecture that every trajectory of $(\Sigma)$ starting in $K$ converges to $\bar{x}$. We prove it but for a restricted set of the problem's parameters.

Theorem 2. 5 Under the following condition $(C)$,

$$
\text { (C) }\left(a_{1}+c\right) \prod_{i=2}^{n-1} \frac{a_{i}}{k_{i}+a_{i}} \leq 1
$$

the system $(\Sigma)$ is globally asymptotically stable in $K$ with respect to $\bar{x}$.

Remark 2. 6 We may express condition $(C)$ in terms of the original parameters, i.e. with $\alpha$ and

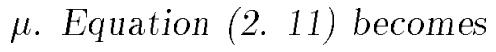

$$
\frac{\alpha}{\mu}\left(a_{1}+c\right) \prod_{i=2}^{n-1} \frac{a_{i}}{\mu k_{i}+a_{i}} \leq 1
$$

It is not surprising that if $\alpha=0$ (i.e. no inhibition) or if $\mu$ is large enough then the condition expressed in (2.12) holds true. 


\section{Proof of Theorem 2. 5}

\subsection{Technical lemmas}

Before starting the proof of the theorem, we establish a series of useful lemmas. We first study the application $z: \mathbb{R}_{+} \rightarrow[0, c)$ by $z(b)=f_{1, b}^{-1}(c)$. We have

\section{Lemma 3. 1}

(i) The application $z$ is strictly increasing from $\mathbb{R}_{+}$to $[0, c)$;

(ii) The application $\varphi_{1} \circ z$ is strictly increasing from $\mathbb{R}_{+}$to $\left[0, \varphi_{1}(c)\right)$ and is concave.

Proof of Lemma 3. 1: The expression of $z(y)$ comes from the equation $\dot{x}_{1}=0$. Differentiating this equation with respect to $y$ results in an expression $z^{\prime}(y)=l\left(z(y), \frac{1}{1+y}\right)$ for some function $l$. We then isolate $\frac{1}{1+y}$ in the equation $\dot{x}_{1}=0$ and substitute the resulting expression into $l$, so that

$$
z^{\prime}(y)=\frac{(c-z(y))^{2}\left(z(y)+k_{1}\right)^{2}}{a_{1}\left(z(y)^{2}+c k_{1}\right)}
$$

and $z^{\prime}(y)>0$ for all $y \geq 0$ because $z(y)<c$ for all $y \geq 0$. Item $(i)$ is proved.

The expressions of $\frac{d \varphi_{1}(z(y))}{d y}$ and $\frac{d^{2} \varphi_{1}(z(y))}{d y^{2}}$ result from straightforward computations:

$$
\begin{gathered}
\frac{d \varphi_{1}(z(y))}{d y}=\varphi_{1}^{\prime}(z) z^{\prime}(y)=\frac{k_{1}(c-z(y))^{2}}{z(y)^{2}+c k_{1}} \\
\frac{d^{2} \varphi_{1}(z(y))}{d y^{2}}=\frac{d}{d z}\left(\frac{d \varphi_{1}(z(y))}{d y}\right) z^{\prime}(y)=-\frac{2 k_{1} c(c-z(y))^{3}\left(z(y)+k_{1}\right)^{3}}{\left(z(y)^{2}+c k_{1}\right)^{3}}
\end{gathered}
$$

so that $\frac{d \varphi_{1}(z(y))}{d y}>0$ and $\frac{d^{2} \varphi_{1}(z(y))}{d y^{2}}<0$ because $z(y)<c$.

Next, we determine, for $b \in[0, c]$, the equilibrium set $E_{b}$ of $(\Sigma)_{b}$, i.e. the set of the equilibrium points of $(\Sigma)_{b}$.

Lemma 3. 2 A point $e \in K$ is an element of $E_{b}$ if and only if its $n$-th coordinate $e_{n}$ is solution in $R_{+}$of the following equation in the unknown $y$

$$
y=M\left(\varphi_{1}(z(y)) \psi(b)\right)
$$

Moreover the previous equation has always solutions and it has exactly one if condition $(C)$ holds.

Remark 3. 3 We chose in this paper to investigate the sets $E_{b}$ 's in an elementary way rather than using the deep work of [4] and [3]. Doing so leads to obtain results on $E_{b}$ which are only valid under 
condition $(C)$ eventhough they are more general. The more complete characterization of the $E_{b}$ 's will appear in the final version of the paper, see [1].

Proof of Lemma 3. 2: if $e \in E_{b}$, then $e_{n}=M\left(\varphi_{1}\left(e_{1}\right) \psi(b)\right)$. Then (3. 4) follows by definition of $z$. When considering the solutions of (3. 4), we are looking for the zeros of the function $h_{b}: \mathbb{R} R_{+} \rightarrow \mathbb{R}$, defined by

$$
h_{b}(y)=y-M\left(\varphi_{1}(z(y)) \psi(b)\right) \text {. }
$$

Note that $h_{b}(0)<0$ and $h_{b}$ tends to $+\infty$ if $y$ tends to $+\infty$ (indeed, $\varphi_{1}(z(y)) \psi(b)$ is bounded). Therefore, if $h_{b}$ is strictly increasing, then $E_{b}$ will have a unique equilibrium point. We show next that this holds true under condition $(C)$.

The derivative of $h_{b}$ is

$$
h_{b}^{\prime}(y)=1-M^{\prime}\left(\varphi_{1}(z(y)) \psi(b)\right) \frac{d \varphi_{1}(z(y))}{d y} \psi(b)
$$

Equation (2. 4) gives an upper bound on $M^{\prime}$ and concavity of $\varphi_{1}(z(y))$ implies that

$$
\frac{d \varphi_{1}(z(y))}{d y}(y) \psi(b) \leq \frac{d \varphi_{1}(z(y))}{d y}(0) \psi(b) .
$$

We also see from (3. 1) and (3. 3) that $\frac{d}{d z}\left(\frac{d \varphi_{1}(z(y))}{d y}\right)<0$ so that

$$
\left.\frac{d \varphi_{1}(z(y))}{d y}\right|_{\mid z=z(0)}<\left.\frac{d \varphi_{1}(z(y))}{d y}\right|_{\mid z=0}=c .
$$

We then have a unique zero to $h_{b}$ if

$$
1-\prod_{i=2}^{n-1} \frac{a_{i}}{k_{i}+a_{i}} c>0
$$

which is satisfied if condition (C) holds. The lemma is proved.

From now on, assume that condition $(C)$ holds. Then, for every $b \geq 0,(\Sigma)_{b}$ has a unique equilibrium point $e(b)$ in $K_{+}$. All the assumptions of Theorem 3.1 of [5] are satisfied. Therefore, $(\Sigma)_{b}$ is globally (with respect to initial states in $K$ ) asymptotically stable with respect to $e(b)$.

The next lemma studies the application $e:[0, c] \rightarrow K_{+}$that associates to $b \in[0, c], e(b)$. Set $e_{n}:[0, c] \rightarrow \mathbb{R}_{+}$for the application that associates to $b$ the $n$-th coordinate of $e(b)$. Note that $\bar{x}_{n}$ is a fixed point of $e_{n}$.

Lemma 3. 4 Assume that condition $(C)$ holds. Then

(1) $e_{n}$ is a strictly decreasing function and $\bar{x}_{n}$ is its unique fixed point; 
(2) if $b_{1}<b_{2}$, then $e\left(b_{2}\right) \ll e\left(b_{1}\right)$;

(3) if $b<\bar{x}_{n}$, then $b<\bar{x}_{n}<e_{n}(b)$ and $\bar{x} \ll e(b)$; similarly if $\bar{x}_{n}<b$, then $e_{n}(b)<\bar{x}_{n}<b$ and $e(b) \ll \bar{x}$.

Proof of Lemma 3. 4: Define $H(w, b):=h_{b}(w)$ for $(y, b) \in R_{+}^{2}$. The differentiation of $H\left(e_{n}(b), b\right)=$ 0 yields

$$
\frac{d e_{n}}{d b}=-\frac{\partial_{b} H}{\partial_{y} H}=\frac{M^{\prime}\left(\varphi_{1}\left(z\left(e_{n}(b)\right)\right) \psi(b)\right) \varphi_{1}\left(z\left(e_{n}(b)\right)\right) \psi^{\prime}(b)}{h_{b}^{\prime}\left(e_{n}(b)\right)}<0,
$$

since $\psi$ is strictly decreasing. In addition, recall that $e_{1}(b)=z\left(\epsilon_{n}(b)\right)$ and $\epsilon_{k}(b)=\varphi_{k}^{-1}\left(f_{k+1}\left(e_{k+1}(b)\right)\right)$ for $2 \leq k \leq n-1$. Then (2) holds since $z$ and the $\varphi_{k}^{-1} \circ f_{k+1}$ 's are strictly increasing functions.

Part (3) is immediate by taking into account the facts that the function $e_{n}$ is strictly decreasing, $\bar{x}_{n}$ is its unique fixed point and (2).

Lemma 3. 5 Consider $\left(I^{l}\right)$ and $\left(S^{l}\right)$ the sequences of points of $K$ defined inductively as follows

$$
\left\{\begin{array}{l}
I^{l+1}=e\left(S_{n}^{l}\right), \\
S^{l+1}=e\left(I_{n}^{l}\right)
\end{array}\right.
$$

with $I^{0}=0$ and $S^{0}$ so that $e(0) \ll S^{0}$ and, for every $x \in T_{c}, x \ll S^{0}$. Here $e(0)$ is the equilibrium point of $(\Sigma)_{0}$. Then, for every $l \geq 0$, we have $I^{l} \ll \bar{x} \ll S^{l}$ and

$$
\lim _{l \rightarrow \infty} I^{l}=\lim _{l \rightarrow \infty} S^{l}=\bar{x},
$$

where $\bar{x}$ is the equilibrium point of $(\Sigma)$.

Proof of Lemma 3. 5: we first prove that for every $l \geq 0, I^{l} \ll \bar{x} \ll S^{l}$, $\left(I^{l}\right)$ is increasing and $\left(S^{l}\right)$ is decreasing with respect to the partial order $\ll$. The argument goes by induction. More precisely, we show by induction that, for every $l \geq 0$, the next proposition $\left(P_{l}\right)$ holds,

$$
\left(P_{l}\right) I^{l} \ll \bar{x} \ll S^{l}, I^{l} \ll I^{l+1}, S^{l+1} \ll S^{l} .
$$

Clearly $\left(P_{0}\right)$ holds. Assume now that $\left(P_{l}\right), l \geq 0$ is true. Then $\bar{x}_{n}<S_{n}^{l}$. By Lemma 3. 4, (3) we have

$$
I^{l+1}=e\left(S_{n}^{l}\right) \ll e\left(\bar{x}_{n}\right)=\bar{x} .
$$

Similarly, we have $\bar{x} \ll S^{l+1}$. Since $I^{l} \ll I^{l+1}$, then $I_{n}^{l}<I_{n}^{l+1}$ and finally

$$
S^{l+2}=e\left(I_{n}^{l+1}\right) \ll e\left(I_{n}^{l}\right)=S^{l+1} .
$$

Similarly, $I^{l+1} \ll I^{l+2}$. 
Since the sequences $\left(I^{l}\right)$ and $\left(S^{l}\right)$ are monotone (componentwise) they converge to $I$ and $S$ with $I \leq \bar{x} \leq S$. By passing to the limit in (3. 7), we have

$$
I=e\left(S_{n}\right), \quad S=e\left(I_{n}\right)
$$

which implies that $I_{n}=e_{n}\left(S_{n}\right)$ and $S_{n}=e_{n}\left(I_{n}\right)$, i.e. $I_{n}$ and $S_{n}$ are fixed points of $e_{n} \circ e_{n}$. The derivative of that function is $\frac{d e_{n}}{d b}\left(e_{n}\right) \frac{d e_{n}}{d b} \geq 0$. If it is smaller than one then $\bar{x}_{n}$ is the unique fixed point of $e_{n} \circ e_{n}$ on $R_{+}$and (3.8) is proved.

Finally to get $\frac{d e_{n}}{d b}\left(e_{n}\right) \frac{d e_{n}}{d b} \leq 1$, it is enough to have $\left|\frac{d e_{n}}{d b}\right| \leq 1$. Taking into account (3. 6), it is enough that, for every $b \in[0, c)$

$$
M^{\prime}\left(\varphi_{1}\left(z\left(e_{n}(b)\right)\right) \psi(b)\right) \varphi_{1}\left(z\left(e_{n}(b)\right)\right)\left|\psi^{\prime}(b)\right| \leq h_{b}^{\prime}\left(e_{n}(b)\right)
$$

which in turn follows from the next inequality

$$
M^{\prime}\left(\varphi_{1}\left(z\left(e_{n}(b)\right)\right) \psi(b)\right)\left(\varphi_{1}\left(z\left(e_{n}(b)\right)\right)+\frac{d \varphi(z(y))}{d y}\left(e_{n}(b)\right) \leq 1 .\right.
$$

Because an upper bound of $M^{\prime}$ is given in equation (2. 4), $\varphi_{1}$ is bounded above by $a_{1}$, and $\frac{d \varphi(z(y))}{d y}$ by $c$, then (3. 11) is implied by condition (C). Since the latter already holds true, the proof of the lemma is finished.

Lemma 3. 6 Let $x \in K$. Then $0 \ll \underline{\lim \gamma_{x}}$

Proof of Lemma 3. 6: Without loss of generality, we take $0 \ll x$ (cf. Proposition 2. 2, (2)) and argue by contradiction. Then there exists $1 \leq i \leq n$ so that $P_{i}$ holds:

$$
\left(P_{i}\right): \quad \forall \varepsilon>0, \forall t>0, \exists t^{\prime}>t, \quad x_{i}\left(t^{\prime}\right)<\varepsilon,
$$

where $x_{j}:=\left(\gamma_{x}\right)_{j}, 1 \leq j \leq n$.

Consider the largest interval $I$ containing $t^{\prime}$ so that $x_{i}(s) \leq \varepsilon$ for $s \in I$. By taking $\varepsilon$ small enough, the interval $I$ is of the type $\left[t_{0}, \cdots\right.$ with $t_{0}>0$. By continuity, $x_{i}\left(t_{0}\right)=\varepsilon$ and $\dot{x}_{i}\left(t_{0}\right) \leq 0$. Clearly, $i$ cannot be equal to 1 , otherwise, from $\dot{x}_{i}\left(t_{0}\right) \leq 0$, we would deduce that $c \leq a_{1} \varepsilon$, which is impossible for $\varepsilon$ small enough.

If $\left(P_{2}\right)$ holds, then $x_{2}\left(t_{0}\right)=\varepsilon$ and $\dot{x}_{2}\left(t_{0}\right) \leq 0$ imply that $x_{1}\left(t_{0}\right) \leq C_{1} \varepsilon$, with $C_{1}$ only depending on the positive parameters $a_{1}, a_{2}, k_{2}, k_{1}$. This means that $\left(P_{1}\right)$ holds and we have a contradiction.

Replacing 2 by any index $i \geq 3$ in the previous sentence indicates that if $\left(P_{i}\right)$ holds, then the same is true for $\left(P_{i-1}\right)$ and by a trivial induction we again have $\left(P_{1}\right)$. Therefore, if any $\left(P_{i}\right)$ holds, we get a contradiction. The proof of Lemma 3. 6 is complete. 


\subsection{Final part of the proof of Theorem 2. 5}

We are now ready to establish Theorem 2. 5. From what precedes, the conclusion is the consequence of the next statement: for every $x \in K$ and for every $l \geq 0$

$$
\left(Q_{l}\right) \quad I^{l} \leq \underline{\lim } \gamma_{x} \leq \varlimsup \lim \gamma_{x} \leq S^{l}
$$

Fix $x \in K$. Proposition $\left(Q_{l}\right)$ is proved inductively. For $l=0$, this is a consequence of Proposition 2. 2, (3). Applying Lemma 3. 6, there exists $\varepsilon>0$ and $t_{0}(\varepsilon)>0$ such that for every $t \geq t_{0}(\varepsilon)$,

$$
\varepsilon v \leq \gamma_{x}(t) \leq S^{0}-\varepsilon v
$$

Then, passing to the limit we have

$$
e\left(S_{n}^{0}-\varepsilon\right) \leq \underline{\lim } \gamma_{x} \leq \varlimsup \lim \gamma_{x} \leq e(\varepsilon)
$$

Note that, in equation (3. 14), $\varepsilon$ may be replaced by any $0<\eta \leq \varepsilon$. Since $e$ is globally Lipschitz over $\mathbb{R}_{+}$, equation (3. 15) implies $\left(Q_{1}\right)$ but also the existence of $t_{1}(\varepsilon)>0$ such that for every $t \geq t_{1}(\varepsilon)$,

$$
I^{1}+C_{1} \varepsilon v \leq \gamma_{x}(t) \leq S^{1}-C_{1} \varepsilon v
$$

for some $0<C_{1} \leq 1$ independant of $\varepsilon$. Notice that equation (3. 16) is of the same type as equation (3. 14) and then leads to equations similar to (3. 15) and again (3. 16). In that way, we obtain, for every $l \geq 2$,

$$
e\left(S_{n}^{l}-C_{l} \varepsilon\right) \leq \underline{\lim } \gamma_{x} \leq \varlimsup \lim \gamma_{x} \leq e\left(I_{n}^{l}+C_{l} \varepsilon\right)
$$

and the existence of $t_{l}(\varepsilon)$ such that, for every $t \geq t_{l}(\varepsilon)$,

$$
I^{l}+C_{l+1} \varepsilon v \leq \gamma_{x}(t) \leq S^{l}-C_{l+1} \varepsilon v
$$

with $C_{l+1} \leq C_{l} \leq 1$ independant of $\varepsilon$. Letting $\varepsilon$ tend to zero in (3. 17), we get $\left(Q_{l}\right)$.

\section{References}

[1] Chitour Y., Grognard F. and Bastin G., "Stability analysis of a metabolic model with sequential feedback inhibition," in preparation.

[2] Coppel W. A., "Stability and asymptotic behavior of differential equations," Heath Math. Mono., D. C. Heath Comp., 1965. 
[3] Li Y. M. and Muldowney J., "Global Stability for the SEIR Model in Epidemiology," Math. Bio. 125 (1995) pp. 155-164.

[4] Mallet-Paret, J. and Smith, Hal L., "The Poincaré-Bendixson theorem for monotone cyclic feedback systems," J. Dynam. Differential Equations 2 (1990), no. 4, pp. 367-421.

[5] Smith H. L., "Monotone Dynamical Systems, An introduction to the theory of competitive and cooperative systems," Math. Surveys and Mono., Vol. 41, AMS, Providence, RI, 1995.

[6] Stephanopoulos G., Aristidou A. and Nielsen J., "Metabolic Engineering: Principles and Metodologies," Academic Press (1997). 